\title{
LEVANTAMENTO DO USO DE PLANTAS MEDICINAIS NA UNIVERSIDADE FEDERAL DO PARANÁ, PALOTINA - PR, BRASIL
}

\author{
Suzana Stefanello ${ }^{1}$ \\ Carina Kozera ${ }^{2}$ \\ Bettina Monika Ruppelt ${ }^{3}$ \\ Daniela Fumagalli ${ }^{4}$ \\ Mariele Pasuch de Camargo ${ }^{5}$ \\ Dircelei Sponciado ${ }^{6}$
}

Resumo: As plantas medicinais representam uma alternativa de tratamento e/ou de prevenção de doenças de grande importância na vida das pessoas, não somente por sua eficácia na ação terapêutica, mas também por se inserir simultaneamente como parte da cultura de um povo. Neste contexto, o presente trabalho teve como objetivo levantar dados sobre a utilização de plantas medicinais pelos funcionários da Universidade Federal do Paraná - Setor Palotina, buscando resgatar informações sobre o seu uso popular para posteriormente subsidiar ações extensionistas com as zeladoras da Universidade. Foram aplicados questionários semiestruturados sobre a utilização de plantas medicinais aos docentes, técnicoadministrativos e funcionários terceirizados responsáveis pela manutenção da estrutura, jardins e da limpeza da Universidade, totalizando 107 entrevistados. Detectou-se que $72 \%$ fazem uso de plantas medicinais, sendo que a maioria utiliza por ser um produto natural e que obtiveram o conhecimento a partir da tradição familiar. A cidreira, hortelã, boldo, camomila, guaco e a marcela foram as plantas citadas como as mais utilizadas, e o resfriado, a gripe, cólicas e a enxaqueca como a doença e/ou mal estar mais frequentemente tratados com plantas medicinais. A partir dos resultados foram planejadas atividades de extensão, como palestras, dinâmicas, práticas e visitas ao horto medicinal da universidade. As atividades foram direcionadas numa primeira fase às funcionárias responsáveis pela limpeza, uma vez que preparavam chás diariamente para os demais envolvidos no estudo. A troca de saberes, a aquisição de novos conhecimentos e a interação entre alunos, professores e o público-alvo envolvido foram os aspectos mais evidentes durante as atividades realizadas, contribuindo para a formação dos alunos e a melhoria da qualidade de vida das pessoas.

Palavras-chave: extensão, conhecimento popular, indicações terapêuticas

\footnotetext{
${ }^{1}$ Docente do Departamento de Biodiversidade, Setor Palotina, UFPR, Rua Pioneiro, 2153, 85950-000 - Palotina, PR, Brasil. E-mail: sstefanello@ufpr.br

${ }^{2}$ Docente do Departamento de Biodiversidade, Setor Palotina, UFPR, Rua Pioneiro, 2153, 85950-000 - Palotina, PR, Brasil. E-mail: carinakozera@yahoo.com.br

${ }^{3}$ Docente do Departamento de Ciências Veterinárias, Setor Palotina, UFPR, Palotina - PR, Brasil

${ }^{4}$ Bióloga, Graduada do Curso de Ciências Biológicas, Setor Palotina, UFPR, Palotina, PR, Brasil

${ }^{5}$ Bióloga, Graduada do Curso de Ciências Biológicas, Setor Palotina, UFPR, Palotina, PR, Brasil

${ }^{6}$ Bióloga, Técnica de Laboratório, Setor Palotina, UFPR, Palotina, PR, Brasil.
} 


\title{
SURVEY OF MEDICINAL PLANTS UTILIZATION AT FEDERAL UNIVERSITY OF PARANA, PALOTINA, PR, BRAZIL
}

\begin{abstract}
Medicinal plants represent an alternative for treatment and/or prevention of major diseases that affect people, not only for its effectiveness in therapeutic action, but also for being simultaneously inserted as a part of people culture. In this context, the objective of the present study was to carry out data on the use of medicinal plants by employees of Federal University of Paraná - Palotina Sector, seeking to retrieve information about its popular use to later subsidize extensionist actions with the University's caretakers. In this study, questionnaires about medicinal plants utilization were applied to professors, outsourced employees responsible for maintaining the UFPR structure, administrative-technicians and outsourced employees responsible for the cleaning, totaling 107 respondents. It was observed that $72 \%$ of the interviewed public have used medicinal plants. Majority of people interviewed also uses medicinal plants considering that they are natural products and based on an obtained knowledge from the family tradition. Among the respondents, lemongrass, mint, boldo, chamomile, guaco and macela were plants cited as the most used. Additionally, cold, flu, colic and migraine were cited as the most frequent diseases to be combated through the use of medicinal plants. From the obtained results, extension activities were planned, such as: lectures, dynamics, technical and practical visits to the University Botanical Garden. In conclusion, the exchange of knowledge, new knowledge acquisition and the interaction among students, professors and target involved audience were the most evident aspects during the carried out activities, contributing to students training and the improvement of people's life quality.
\end{abstract}

Keywords: extension, popular knowledge, therapeutic indications.

\section{INTRODUÇÃO}

A população utiliza diferentes práticas para o tratamento de doenças e sintomas que ameaçam a sua saúde. Apesar do significativo avanço tecnológico e das relevantes melhorias apresentadas na área da saúde nos últimos anos, o uso das plantas medicinais como forma de tratamento complementar é ainda bastante comum. Há alguns anos a Organização Mundial de Saúde (OMS) estimou que aproximadamente $80 \%$ da população mundial já utilizava, e confiava, nos produtos medicinais de origem vegetal (BRANDÃO, 2009; BARNES, ANDERSON, PHILLIPSON, 2012).

O uso de fitoterápicos esteve presente em todas as civilizações e em todos os tempos.

No Brasil, a utilização de plantas medicinais para o tratamento de enfermidades está arraigada às culturas indígena, africana e dos imigrantes europeus (BRANDÃO, 2009).

De forma geral, o acesso aos medicamentos é limitado por questões econômicas, o que leva as pessoas a buscar o tratamento de suas enfermidades e o alívio de seus sintomas

Revista Extensão em Foco, no 15, jan./ jul. (2018), p. 15 - 27. 
utilizando procedimentos mais baratos ou gratuitos, como é o caso da utilização de plantas medicinais retiradas diretamente da natureza (DI STASI, 2007). Além disso, existe grande disposição da população brasileira para utilizar produtos medicinais de origem vegetal. O aumento do uso de medicamentos baseados em plantas medicinais tem refletido no aumento do interesse dos médicos e outros profissionais da saúde (DI STASI, 2007).

Segundo Maciel et al. (2002), o conhecimento popular sobre o uso e a eficácia de plantas medicinais contribuem de forma relevante para a divulgação das potencialidades terapêuticas das espécies e também desperta o interesse de pesquisadores de áreas como a botânica, farmacologia e fitoquímica, enriquecendo com isso o conhecimento sobre as mesmas e intensificando a sua utilização. A eficácia e a segurança, quanto ao uso de muitas plantas medicinais, já foram comprovadas cientificamente o que permitiu seu uso frequente como recurso terapêutico benéfico e indispensável à humanidade (REBOUÇAS, 2009).

Ao longo da história da farmacologia e da medicina, os pesquisadores isolaram compostos obtidos de espécies vegetais de valor medicinal identificados como substâncias básicas para a síntese de centenas de substâncias ativas. Isto ocorreu com base no conhecimento empírico popular e daqueles resgatados nas comunidades tradicionais. Desta forma, foi possível reunir um volume de conhecimentos para a produção de grande parte dos medicamentos disponíveis no mercado, alicerce da medicina moderna (DI STASI, 2007).

Países altamente industrializados têm focado parte de seus investimentos e pesquisas científicas no uso correto das plantas medicinais, visando à extração segura de seus princípios ativos e a comercialização de medicamentos. Segundo Veiga Júnior, Pinto e Maciel (2005) acentua-se diariamente o apelo da mídia para a utilização de produtos naturais nesses países, baseados no argumento de uma vida longa e mais saudável que tais produtos possam oferecer.

As plantas medicinais são consideradas, por significativa parcela da população, como um produto natural isento de produtos químicos, o que contribui para os altos índices de sua utilização. No entanto, apesar de passarem a ser vistas como sinônimos de seguridade e benefício à saúde, é importante ressaltar que muitas delas comumente utilizadas apresentam substâncias capazes de exercer ação tóxica sobre outros organismos vivos (MENGUE, MENTZ, SHENKEL, 2001; von POSER, 2017). Componentes fitoterápicos individuais possuem um amplo conjunto de constituintes químicos (BARNES et al., 2012), sendo, portanto, indispensável conhecer muito bem as plantas que se pretende utilizar, assim como os efeitos adversos que possam causar (FINTELMANN; WEISS, 2010).

Revista Extensão em Foco, nº 15, jan./ jul. (2018), p. 15 - 27. 
Nesse sentido destaca-se o Estado do Paraná, onde a prática do cultivo e do uso de plantas medicinais é bastante evidente (CORRÊA JUNIOR; SCHEFFER, 2004), especialmente na região Oeste devido à tradição regional dos imigrantes italianos e alemães. Por conta disto, nos últimos anos intensificaram-se os estudos e as atividades extensionistas com plantas medicinais promovidas pela Universidade Federal do Paraná (UFPR) do Setor Palotina e as ações da Itaipu Binacional, através do Programa Cultivando Água Boa (RUPPELT et al., 2015), com a finalidade de ampliar o conhecimento e divulgar informações, especialmente sobre a correta identificação das plantas, suas propriedades e sua utilização de forma segura e eficaz.

Diante desse cenário, o presente trabalho teve como objetivo levantar dados sobre a utilização de plantas medicinais pelos funcionários da UFPR - Setor Palotina buscando resgatar informações sobre o uso popular destas plantas para posteriormente subsidiar ações extensionistas com as zeladoras da Universidade.

\section{Materiais e Métodos}

O presente estudo foi realizado entre os anos de 2010 e 2011 por meio do projeto "É hora do chá no Campus Palotina" inserido no Programa de Extensão Universitária "Plantas Medicinais" da UFPR Setor Palotina, registro PROEC (Pró-Reitoria de Extensão e Cultura/UFPR) número 097/10.

A coleta de dados foi realizada por meio de entrevistas, com a aplicação de um questionário elaborado com questões semiestruturadas, objetivando inicialmente avaliar o perfil dos entrevistados, bem como a forma de utilização das plantas medicinais. As entrevistas foram realizadas de forma direta com todos os docentes (49 entrevistados), funcionários técnico-administrativos (33), terceirizados responsáveis pela manutenção da estrutura e dos jardins da UFPR (13) e terceirizadas responsáveis pela limpeza da universidade, aqui denominadas de zeladoras (12), totalizando 107 entrevistados.

Os questionários referiam-se a utilização ou não de plantas medicinais, o modo pelo qual os entrevistados obtiveram os conhecimentos sobre as plantas, quais as finalidades terapêuticas de seus usos e as doenças que acometiam os funcionários com maior frequência.

Após a coleta de dados, as informações foram ordenadas de forma sistematizada em planilhas do programa Microsoft Excel, a partir das quais foram elaborados tabelas e gráficos. Também foi utilizado o programa AMADO - Analyse Graphique d'une Matrice de Données Revista Extensão em Foco, nº 15, jan./ jul. (2018), p. 15 - 27. 
(BERTIN; RISSON; CHAUCHAT, 1994) para a confecção de um gráfico que permite uma análise conjunta dos principais resultados obtidos.

Posteriormente, de posse das informações levantadas, como as principais espécies utilizadas pelos funcionários, para que finalidade e formas de uso utilizadas com mais frequencia, elaborou-se um cronograma de atividades extensionistas como forma de retorno e contribuição ao conhecimento dos entrevistados. A finalidade destas atividades foi a de orientar sobre a identificação das espécies, sobre a forma correta de preparo e manejo de canteiros, de preparo de chás, principais indicações terapêuticas e toxicidade das plantas medicinais. Para estas atividades foram convidadas as zeladoras, uma vez que estavam mais diretamente em contato com as plantas medicinais, através do preparo diário de chás oferecidos aos demais funcionários da instituição.

\section{Resultados e discussão}

A análise dos dados permitiu verificar que $72 \%$ dos entrevistados (77 pessoas) utilizam as plantas medicinais (Figura 1), resultado que condiz com os dados da Organização Mundial de Saúde (OMS). A OMS relata que mesmo com os avanços da medicina moderna grande parte da população dos países em desenvolvimento, tal como o Brasil, depende da medicina tradicional para tratar enfermidades primárias, ressaltando que $85 \%$ desta população busca nessas plantas os cuidados básicos para a sua saúde (BRASIL, 2006).

Dos 107 entrevistados, 55 (51,5\%) eram mulheres, sendo que destas 42 (76,3\%) relataram utilizar as plantas medicinais no cotidiano. No que diz respeito aos homens (52; 48,5\%), 35 (67\%) deles afirmaram utilizá-las (Figura 1). Segundo Marchese et al. (2009), as diferenças de conhecimento relacionadas com o sexo podem ser afetadas por oscilações culturais que refletem na má distribuição de informações sobre as plantas medicinais. Em outras palavras, afirmam que as mulheres herdaram mais dotes culinários e domésticos e, portanto, tais resultados são facilmente compreendidos. Para Oliveira, Oliveira e Andrade (2010) as mulheres demonstram forte interesse pelas plantas medicinais provavelmente por ficarem mais tempo em casa e se responsabilizarem mais com os cuidados com a saúde da família.

Revista Extensão em Foco, nº 15, jan./ jul. (2018), p. 15 - 27. 


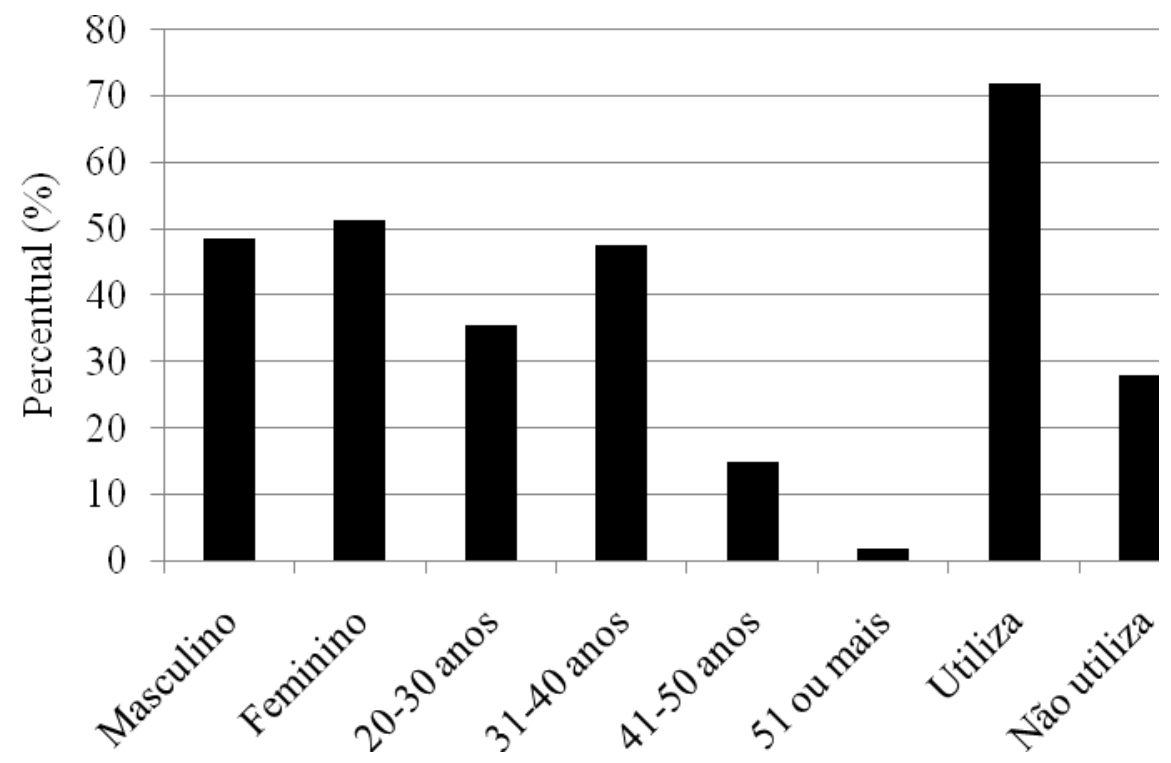

FIGURA 1. Perfil geral e utilização de plantas medicinais pelos entrevistados da UFPR.

Dentre os entrevistados, a maioria possuía faixa etária entre 31 e 40 anos (51 pessoas; $47,6 \%$ ), disse utilizar as plantas medicinais por se referir a um produto natural (47 pessoas; 43,92\%), e que obtiveram o conhecimento sobre estas a partir da tradição familiar (52 pessoas; 48,60\%). Em estudo realizado por Araújo et al. (2014), em uma unidade básica de saúde da família, em Campina Grande - PB, este percentual foi ainda maior (84\% dos entrevistados) sendo relatado que o conhecimento sobre a utilização de plantas medicinais foi adquirido com parentes. O elevado percentual de utilização levantado no presente trabalho pode estar relacionado com a facilidade de obtenção dessas plantas e a alternativa eficaz de qualidade de vida que estas podem oferecer. Além disso, a região Oeste do Paraná é privilegiada em número de espécies, conforme apresentado por Ruppelt et al. (2015). Estes autores listaram e descreveram 43 espécies de plantas utilizadas nessa região com base em levantamentos anteriormente realizados.

De modo similar Viganó, Viganó e Cruz-Silva (2007) e Paulert et al. (2014) também relataram que a maior parte dos seus entrevistados (36 e 74\%, respectivamente) faz uso das plantas medicinais por ser um produto natural. Para Diniz et al. (1997) a utilização de fitoterápicos envolve questões de cidadania, pois ao utilizar plantas medicinais o cidadão se torna agente ativo nos cuidados de sua saúde e não apenas mais um paciente nas mãos dos profissionais que atuam através da indicação de medicamentos alopáticos. Porém, muitas vezes por considerarem as plantas medicinais um produto natural, muitas pessoas acreditam Revista Extensão em Foco, nº 15, jan./ jul. (2018), p. 15 - 27. 
que as mesmas não causam dano a saúde, o que foi recentemente constatado por Araújo et al. (2014). Portanto, ações que levem ao esclarecimento da população quanto aos possíveis riscos da utilização indiscriminada e da automedicação são de fundamental importância.

No estudo foram indicados 16 diferentes problemas de saúde tratados com plantas medicinais pelos entrevistados (Figura 2). O grupo dos docentes do sexo feminino foi o que citou o maior número de problemas de saúde (11), seguido pelo grupo dos técnicoadministrativos do sexo masculino, que citou nove problemas de saúde. As zeladoras e os docentes do sexo masculino citaram oito problemas e o grupo dos funcionários da manutenção seis problemas de saúde. A gripe e o resfriado foram os mais citados por todos os grupos e resultaram nos maiores percentuais de indicações terapêuticas. Em seguida, destacou-se a enxaqueca (que não foi citada apenas pelo grupo dos docentes do sexo masculino), insônia, cólicas e gastrite.

Neste estudo observou-se uma maior indicação das plantas medicinais para uso de problemas relacionados ao sistema respiratório, no caso gripes e resfriados, que acomete entrevistados dos diferentes grupos, faixas etárias e sexo. Para tais problemas inclusive, o guaco foi introduzido nos canteiros da universidade. Segundo Castro et al. (2005), Mikania glomerata (guaco) possui reconhecidamente propriedades broncodilatadoras e expectorantes e, portanto, é indicada no combate a tosse, asma, bronquite, ronquidão e outros sintomas associados a gripes e resfriados.

Em trabalho semelhante a este, desenvolvido por Viganó, Viganó e Cruz-Silva (2007), as plantas medicinais foram indicadas principalmente para doenças do sistema respiratório e digestório, seguidas de doenças do sistema circulatório.

Revista Extensão em Foco, nº 15, jan./ jul. (2018), p. 15 - 27. 


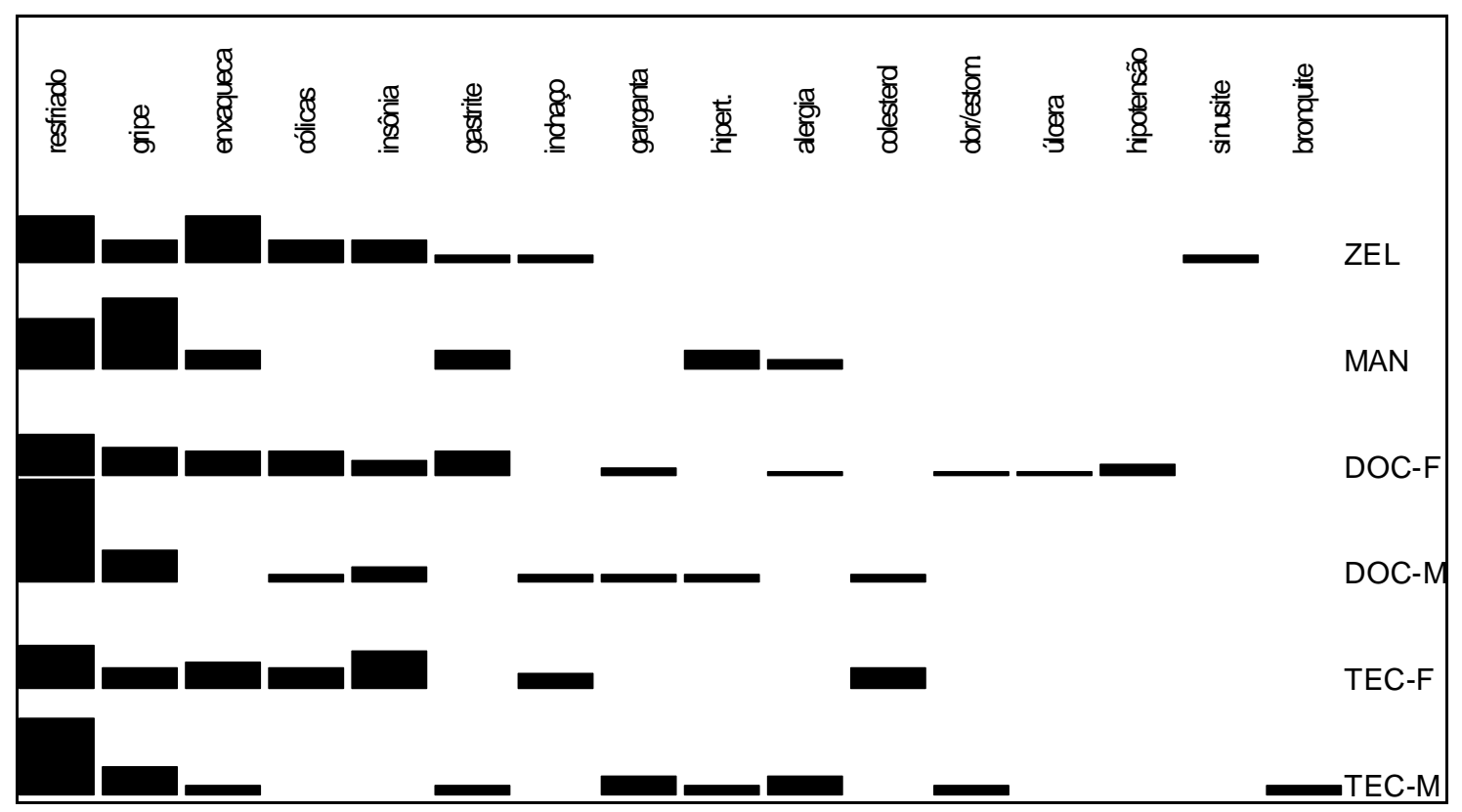

FIGURA 2. Problemas de saúde indicados pelos entrevistados da UFPR e tratados com plantas medicinais. A altura das colunas verticais indica a quantidade de vezes que foi citada no grupo. $\mathrm{ZEL}=$ zeladoras; $\mathrm{MAN}=$ funcionários terceirizados responsáveis pela manutenção da estrutura e jardins; $\quad$ DOC $=$ docentes, M-masculino, F-feminino; $\quad T E C=$ técnicos administrativos, M-masculino, F-feminino.

Outro problema de saúde citado, que esteve entre os de maior indicação terapêutica, foi a cólica, citada principalmente por entrevistados do sexo feminino, tanto no grupo de docentes, técnico-administrativos como das zeladoras. Possivelmente esteja relacionada aos problemas do sistema reprodutor (cólicas menstruais).

Com relação às plantas, foram citadas 50 diferentes espécies utilizadas para fins medicinais, algumas delas, inclusive, muito comumente utilizadas na culinária como temperos (por exemplo: alho, cebola, salsinha), na preparação de saladas ou refogados (por exemplo: alface, chicória, couve) e condimentos (por exemplo: canela, gengibre). Dentre as plantas citadas, a camomila, a cidreira, o boldo, a hortelã, o guaco, a macela, a erva-doce, a malva, a quebra-pedra, o alecrim e a carqueja foram listados por entrevistados de todos os grupos e estão entre as plantas mais utilizadas por estas categorias de entrevistados (Quadro 1; Figura 3). O boldo, a erva-cidreira e a hortelã, além do capim-santo, também aparecem entre as plantas mais citadas pelos entrevistados por Araújo et al. (2014) em levantamento realizado em uma unidade de saúde da família.

A utilização da camomila pode estar relacionada ao fato de apresentar propriedades fitoterápicas digestivas e sedativas; a cidreira, é especialmente empregada pelo sabor 
agradável e por sua ação calmante; a hortelã especialmente para problemas do sistema respiratório (como gripes, descongestionante nasal) e digestório. O boldo é utilizado para males do fígado e problemas de digestão; e o guaco, que tem usos caseiros diversos, tem ação comprovada apenas sobre o sistema respiratório agindo como broncodilatador, antitussígeno e expectorante. $\mathrm{O}$ alecrim tem indicações aceitas internacionalmente especialmente para tratamento caseiro de casos de hipertensão, problemas digestivos, perda de apetite e externamente para sintomas de reumatismo; e a macela é utilizada principalmente para problemas do sistema digestório, cólicas de origem nervosa e menstruais e como sedativo (LORENZI; MATOS, 2008). Os principais usos dessas plantas, citadas como as de maior utilização pelos entrevistados, coincide, em parte, com as principais doenças listadas na entrevista, que foram as gripes e resfriados, enxaqueca, cólicas e insônia.

Quadro 1. Plantas medicinais mais citadas nas entrevistas realizadas.

\begin{tabular}{|l|l|l|}
\hline \multicolumn{1}{|c|}{ PLANTA MEDICINAL } & \multicolumn{1}{|c|}{ FAMÍLIA } & \multicolumn{1}{c|}{ ESPÉCIE } \\
\hline Alecrim & Lamiaceae & Rosmarinus officinalis L. \\
\hline Boldo & Lamiaceae & Plectranthus barbatus Andr. \\
\hline Camomila & Asteraceae & Chamomilla recutita (L.) Rauschert \\
\hline Carqueja & Asteraceae & Baccharis crispa Spreng. \\
\hline Cidreira & Poaceae & Cymbopogon citratus (DC.) Stapf \\
\hline Erva-doce & Apiaceae & Pimpinella anisum L. \\
\hline Guaco & Asteraceae & Mikania glomerata Spreng. \\
\hline Hortelã & Lamiaceae & Mentha spp. \\
\hline Macela & Asteraceae & Achyrocline satureoides (Lam.) DC. \\
\hline Malva & Malvaceae & Malva sylvestris L. \\
\hline Quebra-pedra & Phyllanthaceae & Phyllanthus niruri L. \\
\hline
\end{tabular}

Além do uso medicinal, foram formuladas perguntas para avaliar o uso de plantas medicinais como temperos e condimentos no cotidiano das pessoas entrevistadas, isso porque boa parte delas geralmente tem uso atribuído a essas duas finalidades, que seria o tratamento da saúde e a culinária. No total, foram listadas 24 espécies, das quais a cebolinha, o louro, o manjericão, o orégano e a salsinha foram listados por todos os grupos entrevistados. Observou-se que dez das espécies listadas como de uso condimentar ou tempero também foram relacionadas na lista de uso medicinal, evidenciando o seu uso para diferentes finalidades, a saber: alecrim, alfavaca, alho, canela, cebola, gengibre, hortelã, nozmoscada, salsinha e sálvia.

Revista Extensão em Foco, nº 15, jan./ jul. (2018), p. 15 - 27. 


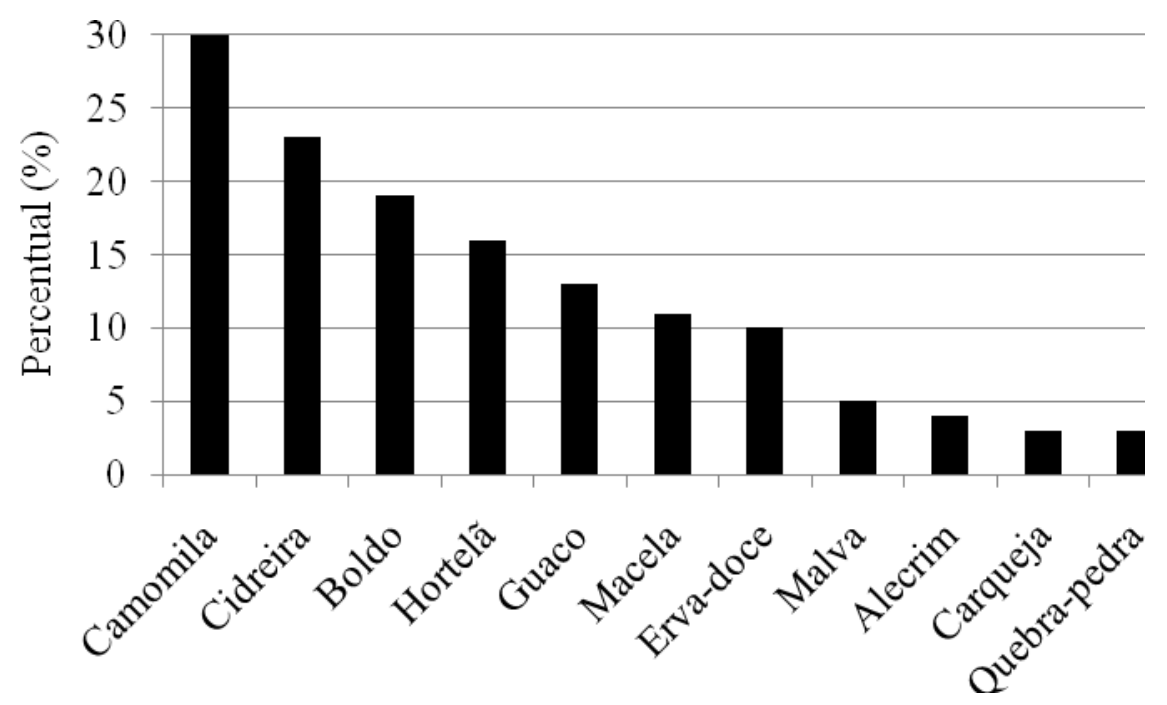

FIGURA 3. Percentual de utilização das plantas medicinais mais citadas pelos entrevistados da UFPR.

Com os dados obtidos a partir dos questionários, e após as análises apresentadas, foram ministradas diferentes atividades extensionistas com as zeladoras da universidade (funcionárias tercerizadas responsáveis pela limpeza da UFPR). Esse grupo foi escolhido, dentre os entrevistados, pelo fato de estarem em contato com as plantas medicinais do horto da universidade no preparo diário de chás oferecidos para os demais envolvidos no estudo. As atividades realizadas envolveram palestras sobre temas relacionados às formas de cultivo das espécies, coleta, armazenamento, diferenças entre nome científico e popular, principais formas de preparo dos chás, indicações terapêuticas, orientações e principais cuidados. Além disso, foram feitas atividades práticas envolvendo visitas ao Horto de Plantas Medicinais e Aromáticas da UFPR Setor Palotina, para o conhecimento das plantas; dinâmicas de grupo no Laboratório de Botânica, para a pesagem de plantas medicinais frescas e secas e com isso o entendimento das suas diferentes formas de consumo; e ainda, para finalizar, foi realizado um concurso de receitas preparadas com algumas espécies de plantas medicinais. Esta atividade, de caráter lúdico, teve a intenção de ser um momento de descontração e ao mesmo tempo de troca de saberes entre as zeladoras, professores e alunos extensionistas.

Segundo relatos das participantes, as atividades possibilitaram o esclarecimento de dúvidas e a aquisição de novos conhecimentos. Isso permitiu ainda que o preparo dos chás oferecidos para os demais funcionários da universidade fosse realizado de forma mais segura,

Revista Extensão em Foco, nº 15, jan./ jul. (2018), p. 15 - 27. 
evitando, por exemplo, que plantas como a citronela (Cymbopogon winterianus), que são morfologicamente parecidas com plantas do capim-limão (Cymbopogon citratus) fossem utilizadas erroneamente no preparo dos chás. Por meio destas ações, as informações recebidas certamente serão multiplicadas em suas famílias e, consequentemente na comunidade.

Além da troca de conhecimentos, as atividades também permitiram uma maior aproximação da universidade com a comunidade e a integração entre os envolvidos, contribuindo na formação acadêmica e cidadã dos alunos de graduação, transpondo, dessa forma, os limites das salas e das aulas convencionais.

\section{Conclusões}

A partir deste estudo foi possível concluir que as plantas medicinais continuam em lugar de destaque em nossa sociedade. A região Oeste do Paraná é privilegiada em número de espécies conforme constatado na lista de plantas citadas nas entrevistas e altos percentuais de uso no cotidiano dos entrevistados. O consumo foi indicado pela fácil obtenção e menor custo do que os medicamentos industrializados. Ressalta-se, porém, que não devem ser utilizadas em substituição a esses sem prescrição médica.

Além disso, cabe ressaltar que o uso das plantas medicinais deve ser feito de maneira segura, já que grande parte das plantas conhecidas e utilizadas não passaram por estudos e pesquisas detalhadas que revelem suas contra indicações e efeitos colaterais.

Com o presente trabalho, verificou-se que a maioria dos entrevistados faz uso das plantas por se referir a um produto natural, sem considerar o fato de que podem oferecer graves riscos à saúde quando não utilizadas de maneira correta.

O presente levantamento permitiu analisar diferentes fatores no que diz respeito ao uso das plantas medicinais no convívio acadêmico da UFPR e permitiu a realização de atividades extensionistas direcionadas a um dos grupos entrevistados resultando numa troca de saberes, atualização e aquisição de novos conhecimentos.

\section{Referências}

ARAÚJO, C.R.F.; SILVA, A.B.; TAVARES, E.C.; COSTA, E.P.; MARIZ, S. Perfil e prevalência de uso de plantas medicinais em uma unidade básica de saúde da família em Campina Grande, Paraíba, Brasil. Revista de Ciências Farmacêuticas Básica e Aplicada, v. 35, n. 2, p. 233-238, 2014.

Revista Extensão em Foco, nº 15, jan./ jul. (2018), p. 15 - 27. 
BRASIL. Ministério da Saúde. Política nacional de plantas medicinais e fitoterápicos. Ministério da Saúde, Secretaria de Ciência, Tecnologia e Insumos Estratégicos, Departamento de Assistência Farmacêutica. Série B. Textos Básicos de Saúde. Brasília: Ministério da Saúde, 2006. $60 \mathrm{p}$.

BERTIN, J.; RISSON, J.; CHAUCHAT, J.H. AMADO - Analyse Graphique d'une Matrice de Données. Editeur CISIA, France. 1994.

BARNES, J.; ANDERSON, L.A.; PHILLIPSON, J.D. Fitoterápicos. 3. ed. Porto Alegre: Artmed, 2012. 720p.

BRANDÃO, D.C. A história da fitoterapia no Brasil. In: LIMA, S. M. R. R. Fitomedicamentos na prática ginecológica e obstétrica. 2 ed. São Paulo: Atheneu, 2009. p.1-11.

CASTRO, E. M.; PINTO, J.E.; MELO, H.C.; SOARES, A.M.; ALVARENGA, A. A.; LIMA JÚNIOR, E. C. Aspectos anatômicos e fisiológicos de plantas de guaco submetidas a diferentes fotoperíodos. Horticultura Brasileira, Brasília, v.23, n.3, p.846-850, jul./set. 2005 .

CORRÊA JUNIOR, C.; SCHEFFER, M.C. Produção de plantas medicinais, condimentares e aromáticas no Estado do Paraná. In: CORRÊA JUNIOR, C.; GRAÇA, L. R.; SCHEFFER, M.C. Complexo agroindustrial das plantas medicinais, aromáticas e condimentares no Estado do Paraná - diagnóstico e perspectivas. Curitiba: Sociedade Paranaense de Plantas Medicinais Emater/Embrapa Florestas, p. 48-68, 2004.

DINIZ, M.F.F.; OLIVEIRA, R.A.G.; MEDEIROS, A.C.D.; MALTA JÚNIOR, A. Memento fitoterápico: as plantas como alternativa terapêutica, conhecimentos populares e científicos. João Pessoa: Editora Universitária / UFPB, 1997. 205 p.

DI STASI, L. C. Plantas medicinais: verdades e mentiras. São Paulo, UNESP, 2007. 133 p.

FINTELMANN, V.; WEISS, R. F. Manual de fitoterapia. 11 ed., Rio de Janeiro: Guanabara Koogan, 2010. 526p.

LORENZI, H.; MATOS, F.J.A. Plantas Medicinais no Brasil: nativas e exóticas. 2. ed. Nova Odessa: Instituto Plantarum, 2008. 560p.

MACIEL, M.A.M.; PINTO, A.C.; VEIGA JUNIOR, V.F.; GRYNBERG, N.F.; ECHEVARRIA, A. Plantas medicinais: a necessidade de estudos multidisciplinares. Química Nova, v.25, n.3, p.429-438, 2002.

MARCHESE, J. A.; MING, L.C.; FRANCESCHI, L.; CAMOCHENA, R.C.; GOMES, G. D.R.; PALADINI, M.V.; CAPELIN, D.; MARCHESE, C. F. Medicinal plants used by "Passo da Ilha" rural community in the city of Pato Branco, southern Brazil. Anais da Academia Brasileira de Ciências, v.81, n.4, p. 691-700, 2009. 
MENGUE, S.S.; MENTZ, L.A.; SCHENKEL, E.P. Uso de plantas medicinais na gravidez. Revista Brasileira de Farmacognosia, v. 11, n. 1, p. 21-35, 2001.

OLIVEIRA, G.L.; OLIVEIRA, A.F.M., ANDRADE, L.H.C. Plantas medicinais utilizadas na comunidade urbana de Muribeca, Nordeste do Brasil. Acta Botanica Brasilica, v. 24, n. 2, p. 571-577, 2010.

PAULERT, R. PUJARRA, S.; OLIVEIRA, F.G.; ZONETTI, P.C.; RUPPELT, B.M. Utilização popular de plantas medicinais nos clubes de mães de Palotina - PR. Ciência em Extensão, v.10, n. 2, p.55-64, 2014.

REBOUÇAS, F.S. Cultivo in vitro de plantas medicinais: Ocimum basilicum L. e Cissus sicyoides L. Dissertação (Mestrado) - Universidade Federal do Recôncavo da Bahia. Curso Ciências Agrárias, Ambientais e Biológicas. Área de concentração Fitotecnia. Cruz das Almas, 2009, 61f.

RUPPELT, B.M.; KOZERA, K.; ZONETTI, P. C.; PAULERT, R.; STEFANELLO, S. Plantas medicinais utilizadas na região Oeste do Paraná. Curitiba: UFPR, 2015. 126 p.

VEIGA JUNIOR, V. F.; PINTO, A. C.; MACIEL, M. A. M. Plantas medicinais: cura segura? Química Nova, Rio de Janeiro, v. 28, n.3, p. 519-528, 2005.

VIGANÓ, J.; VIGANÓ, J. A.; CRUZ-SILVA, C. T. A. Utilização de plantas medicinais pela população da região urbana de Três Barras do Paraná. Acta Scientiarum, Health Sciences, v. 29, n.1, p. 51-58, 2007.

VON POSER, G. L. A quimiotaxonomia na sistemática dos seres vivos. In: SIMÕES, C.M.O.; SCHENKEL, E. P.; MELlO, J. C. P.; MENTZ, L. A.; PETROVICK, P. R. Farmacognosia: do produto natural ao medicamento. Porto Alegre: Artmed, p. 23-28, 2017.

Revista Extensão em Foco, nº 15, jan./ jul. (2018), p. 15 - 27. 